\title{
Cytotoxic Effect of Protoporphyrin IX to Human Leukemia U937 Cells under Ultrasonic Irradiation
}

\author{
Yixiang Li $\mathrm{L}^{\mathrm{a}}$ Xiaomin Su ${ }^{\mathrm{a}}$ Xiaobing Wang ${ }^{\mathrm{a}}$ Albert Wingnang Leung ${ }^{\mathrm{b}}$ \\ Chuanshan Xub Pan Wang ${ }^{a, b}$ Quanhong Liu ${ }^{a}$
}

aKey Laboratory of Medicinal Resources and Natural Pharmaceutical Chemistry, Ministry of Education, National Engineering Laboratory for Resource Developing of Endangered Chinese Crude Drugs in Northwest of China, College of Life Sciences, Shaanxi Normal University, Xi'an, bSchool of Chinese Medicine, Faculty of Medicine, The Chinese University of Hong Kong, Shatin, Hong Kong, China

\section{Key Words}

Sonodynamic therapy $\cdot$ Protoporphyrin IX $\cdot$ Apoptosis $\cdot$ Reactive oxygen species $\cdot$ Human leukemia U937 cells

\begin{abstract}
Background: Sonodynamic therapy (SDT) is an alternative strategy that manages malignancies via the generation of cytotoxic factors during ultrasound-activated sono-sensitive agents. However, the detailed mechanisms are not clear. This study was to identify the cytotoxic effects of ultrasound-activated protoporphyrin IX (PpIX) on U937 cells. Methods: Flow cytometry was performed to detect the time course for PpIX uptake in U937 cells. Sub-cellular localization of PpIX in U937 cells was visualized by inverted confocal laser scanning microscope. Following PpIX-mediated SDT treatment, cell viability was evaluated by the 3-(4, 5- dimethylthiazol2-yl)-2, 5-diphenyltetrazoliumbromide (MTT) assay; nuclear damage was observed under fluorescent microscope; DNA fragmentation and mitochondrial membrane potential disruption were measured by flow cytometry. The role of reactive oxygen species (ROS) in SDT-induced cell death was also evaluated. Results: We observed that PpIX is mainly localized in the mitochondria, with a maximal uptake within $2 \mathrm{~h}$. Compared with PpIX or ultrasound alone, PpIX plus ultrasound treatment significantly declined cell viability, caused more serious damage of cell morphology, DNA and mitochondria. In the combined treatment group, the intracellular ROS was greatly higher than in other groups; ROS scavenger $\mathrm{N}$-acetylcysteine could effectively rescue the loss of mitochondria membrane potential and cell viability induced by SDT. Conclusion: Taken together, these findings primarily indicated that fatal damage could be induced by PpIX-mediated SDT in U937 cells, and the intracellular ROS was involved during this process.




\section{Introduction}

Sonodynamic therapy (SDT) is a relatively new and promising cancer treatment modality which involves the synergistic effect on cell killing by the combination of sonosensitizers and ultrasound $[1,2]$. Ultrasound, especially focused ultrasound has an ability in focusing energy into small volumes, with minimal undesirable side effects [3, 4]. Sonosensitizers can preferentially accumulate in malignant cells and be activated by ultrasound of appropriate property [5]. Several in vitro and in vivo studies have confirmed that SDT has significant damage effects on various tumors, and shows great potential for target cancer therapy $[2,4$, $6,7]$.

Protoporphyrin IX (PpIX) is known as an effective component of hematoporphyrin derivatives, which was found preferentially accumulated in rapid proliferating cancer cells, thus providing selective destruction of tumors exposed to ultrasound [8]. Under normal condition, PpIX and heme (iron-PpIX) are vital for cellular homestasis, but free uncommitted pools of these molecules are highly cytotoxic [9]. Studies have found that the concentration of PpIX in cell or tissue could be increased not only by administration of its precursor $\delta$-aminolevulinic acid (5-ALA) but also PpIX itself [10-12]. Obviously, the exogenous PpIX leads to more visible cytotoxicity than endogenous PpIX in SDT [10].

Many studies have documented SDT induced apoptosis in a variety of cell types by an array of different sensitizers [1-7]. Apoptosis induction is arguably the most potent defense against cancer. The process of apoptosis induced by SDT may involve SDT induced damage on several cellular components, such as plasma membrane, mitochondria, nucleus and chromatin. On the other hands, some observations indicate that SDT may induce tumor cell apoptosis through the influence of genes regulating apoptosis [4, 12-14]. However, since the efficacy of SDT may be influenced by multiple factors, including the nature of the biological model, the sonosensitizer, and the ultrasonic parameters, the detailed mechanism of SDT is unclear until now. Therefore, this study addressed the sonodynamic damaging effect of PpIX combined with ultrasound on human leukemic cell line U937.

\section{Materials and Methods}

Sonosensitizer and chemicals

PpIX was purchased from Sigma Chemical Company and the purity was greater than $95 \%$. PpIX was dissolved in deionized water at a stock concentration of $1 \mathrm{mg} / \mathrm{ml}$, sterilized and stored in the dark at $-20{ }^{\circ} \mathrm{C}$ prior to use. Mito Tracker Green (MTG) was purchased from Molecular Probes Inc. (USA). N-acetylcysteine (NAC), 3-(4, 5-dimethylthiazol-2-yl)-2, 5-diphenylter-trazolium bromide tetrazolium (MTT), 4', 6-diamidino2-phenylindole dihydrochloride (DAPI), propidium iodide (PI), Rhodamine 123 (Rh 123) and 2, 7-DCFdiacetate (DCFH-DA) were from Sigma-Aldrich (USA). All other reagents were commercial products of analytical grade.

\section{Cell line and culture}

Human leukemic cell line U937 was obtained from Institute of Chinese Academy of Medical Sciences, Beijing, China. Cells were grown in RPMI 1640 medium (Gibco) supplemented with $10 \%$ fetal bovine serum (Gibco), $100 \mathrm{U} / \mathrm{ml}$ penicillin, $100 \mu \mathrm{g} / \mathrm{ml}$ streptomycin, and $2 \mathrm{mM}$ L-glutamine under a humidified $5 \%$ carbon dioxide $\left(\mathrm{CO}_{2}\right)$ and $95 \%$ air atmosphere at $37{ }^{\circ} \mathrm{C}$. Cells in the exponential phase were used for experiment, and the cell viability was above $95 \%$ using trypan blue exclusion test before each treatment.

\section{Ultrasonic exposure system}

The experimental set-up for insonation was similar as previously described [13]. Briefly, focused ultrasound transducer with a circular ceramic aperture of $15 \mathrm{~mm}$ in diameter, manufactured by the Institution of Applied Acoustics, Shaanxi Normal University (Xi'an, China), was submerged in degassed water in the tank facing directly upward. The electrical signal was generated and amplified by a multi-functional generator (AG1020, T\&C Power Conversion, Inc., Rochester, NY, USA) before being sent to the transducer. 
The ultrasound field distribution was recorded by the Ultrasonics C-Scan image System (3560C, Physical acoustics corporation, USA). The central focal spot was shown at about $26 \mathrm{~mm}$ away from the piezoelectric sound source. The produced focal area was about $6-8 \mathrm{~mm}$ in the lateral dimension and $1.6 \mathrm{~cm}$ in the axial direction. Ultrasound irradiation was conducted with a frequency of $1.1 \mathrm{MHz}$ in a continuous wave mode for $60 \mathrm{~s}$ duration. $0.5 \mathrm{ml}$ sample contained in a polypropylene test tube was placed into the center of the focal zone for irradiation. Samples were submerged entirely in degassed water and the test tube was rotated at $20 \mathrm{rpm}$ by a micro-motor to improve mixing and to provide a uniform exposure. Every tube was capped by a rubber stopper as a sound absorber to minimize standing wave effects, but this process was not particularly efficient because there was air between the cap and suspension; therefore, the sonication setup used in this study could have resulted in some standing waves. Damage to the U937 cells from ultrasound treatment was evaluated in this state.

Due to the difficulty in measuring the acoustic intensity in the field, we measured it in the free field instead. Suppose in the plane wave conditions and the ultrasound attenuation was negligible, the peak acoustic pressure $(p(t))$ at the central focus of the sound field $(-6 \mathrm{~dB})$ was measured using a needle broadband hydrophone (PT-080546, active element size: $0.8 \mathrm{~mm}$, Institute of Acoustics of Chinese Academy of Sciences, China), and the detected signal was registered on a digital oscilloscope (Tektronix, Inc., USA). The acoustic peak intensity calculated from the eqution $I(t)=p^{2}(t) / \rho c$, where $(\rho)$ is the density of the medium, and (c) is the speed of sound in the medium. The spatial average value was evaluated using a three dimensional micromanipulator scanning system, and the temporal - average value was calculus analyzed as a function of the duration time. During $60 \mathrm{~s}$ ' exposure, the acoustic intensity in the exposed area was calculated as about $1.0 \mathrm{~W} / \mathrm{cm}^{2}\left(\mathrm{I}_{\text {SATA }}\right.$, spatial average time average intensity) when the load power by the AG1020 apparatus indicated $3 \mathrm{~W}$.

For all experiments, the coupling water was degassed before ultrasound treatment and was maintained at room temperature during irradiation. Temperature increase inside the test tube was measured before and after ultrasound treatment with a digital thermometer, and no significant variation of temperature was detected $\left( \pm 1^{\circ} \mathrm{C}\right)$. Thus, any bio-effects observed in this study were considered to be non-thermal.

\section{SDT treatment protocol}

U937 cells $\left(1 \times 10^{6}\right.$ cells $\left./ \mathrm{ml}\right)$ in the exponential phase were collected and divided randomly into four groups: (1) control; (2) PpIX alone; (3) ultrasound alone (Us); (4) ultrasound plus PpIX (SDT). The cells in the PpIX alone and SDT groups were incubated with PpIX, while an equivalent quantity of medium was added to the control and Us groups instead of PpIX. The cells in the Us and SDT groups were exposed to ultrasound at a frequency of $1.1 \mathrm{MHz}$ and an intensity of $1 \mathrm{~W} / \mathrm{cm}^{2}$ for $60 \mathrm{~s}$ duration. After the treatment procedure, cells were re-suspended in fresh medium and cultured for an additional time as specified in the text and then subjected to different analysis. During the experiments, all the samples were placed in the dark to avoid the light-activated impact as much as possible. For inhibitory studies, $5 \mathrm{mM}$ special reactive oxygen species (ROS) scavenger NAC was added to culture medium prior to loading PpIX $1 \mathrm{~h}$. The inhibitor at the used concentrations did not yield any significant damage to U937 cells.

\section{Estimating cellular uptake and localization of PpIX}

U937 cells $\left(1 \times 10^{6}\right.$ cells $\left./ \mathrm{ml}\right)$ were incubated with PpIX at various concentration $(0,0.5,1,5,10 \mu \mathrm{g} / \mathrm{ml})$ for $0.5,1,2,3,4,5$ and $6 \mathrm{~h}$. Cells were harvested and washed three times with phosphate-buffered saline (PBS, pH 7.2). PpIX autofluorescence was measured by flow cytometer (Guava easyCyte 8HT, Millipore Co.). A total of 5000 cells were analyzed for each sample, and the mean fluorescence intensities were recorded to represent the uptake of PpIX by U937 cells indirectly.

For detecting the cellular localization of PpIX, U937 cells were incubated with $2 \mu \mathrm{g} / \mathrm{ml}$ PpIX for $2 \mathrm{~h}$, then co-loaded with 3 nM MTG. Subsequently, cells were washed with PBS and imaged using inverted confocal laser scanning microscope (TCS SP5, Leica Co.). In multi-channel imaging, photomultiplier sensitivities and offsets were set to a level at which bleed through effects from one channel to another were negligible.

\section{Cell viability assessment}

The cell viability was evaluated by MTT assay to reflect the effect of ultrasound on the viability of U937 cells in the presence and absence of PpIX. The PpIX at concentration of $2 \mu \mathrm{g} / \mathrm{ml}$ was added to PpIX alone group and SDT group. After different treatment, cells were harvested and $100 \mu \mathrm{l}$ suspension in each 


\begin{tabular}{|c|c|c|}
\hline Cellular PI & Cell Physiol Biochem 2014;33:1186-1196 & \\
\hline and Biochemistry & $\begin{array}{l}\text { DOI: 10.1159/000358687 } \\
\text { Publisned onine: April I5, } 2014\end{array}$ & $\begin{array}{l}\text { O } 2014 \text { S. Karger AG, Basel } \\
\text { www.karger.com/cpb }\end{array}$ \\
\hline
\end{tabular}

group were added to 96 well culture plates, and the cell viability was determined at $4 \mathrm{~h}$. Briefly, $10 \mu \mathrm{l}$ MTT solution (5 mg/ml in PBS) was added to each well and the mixture was incubated for additional $4 \mathrm{~h}$ at 37 ${ }^{\circ} \mathrm{C}$ in a $\mathrm{CO}_{2}$ incubator. The formed formazan crystals were dissolved in the combined solution buffer (10 $\%$ SDS, 5\% isobutyl alcohol, $0.01 \mathrm{M} \mathrm{HCl}$ ) and the absorbance at $570 \mathrm{~nm}$ was recorded using a microplate reader (ELX800, Bio-Tek) against the reference value at $630 \mathrm{~nm}$. The cell viability of treated samples was then analyzed by comparing to the incubated but non-treated control.

\section{Morphological observation}

In order to detect changes in nuclei morphology of U937 cells after SDT treatment, DAPI staining was performed as previous described [15]. DAPI is a fluorescence probe, which binds to natural doublestranded DNA, and representative the change of the nuclei morphology. At $4 \mathrm{~h}$ post treatment, U937 cells were harvested and washed three times with PBS and then stained with $4 \mu \mathrm{g} / \mathrm{ml}$ DAPI at $37{ }^{\circ} \mathrm{C}$ for 15 minutes. Then the stained cells were washed and re-suspended with PBS and mounted on slides. Cells were visualized under a fluorescence microscope (Nikon) with standard excitation filters. Excitation wavelength was $364 \mathrm{~nm}$ and emission wavelength was $454 \mathrm{~nm}$. Simultaneously, the corresponding phase contrast images were recorded.

\section{Analysis of DNA fragmentation}

The detection of DNA fragmentation by flow cytometry was performed through adding PI to the cells and permeabilizing them by freeze-thawing [16]. PI is an intercalating agent and a fluorescent molecule, which can bind to double-stranded DNA molecule and shows red fluorescence. Once DNA fragments increase, the fluorescence intensity of PI would be reduced. At $4 \mathrm{~h}$ post treatment, cells in each treatment group were harvested and washed with PBS, then re-suspended in $200 \mu \mathrm{l}$ Hanks' buffer containing $5 \mu \mathrm{g} /$ $\mathrm{ml}$ PI in $1.5 \mathrm{ml}$ eppendorf tube. The tubes were immediately placed in liquid nitrogen for $30 \mathrm{~s}$ and then thawed in $37^{\circ} \mathrm{C}$ for 5 minutes. This procedure permeabilizes the cells and stains them with PI. Samples were analyzed by flow cytometry. Histograms were analyzed using FCS Express V3 (Novo Tech Software Ltd.).

\section{Examination of mitochondrial membrane potential $\left(\Delta \psi_{m}\right)$}

Rhodamine $123(\mathrm{Rh} 123)$ was widely used to evaluate perturbation in mitochondria membrance potential $\left(\Delta \psi_{\mathrm{m}}\right)$ as previously described [17]. At $2 \mathrm{~h}$ after different treatment, cells were harvested, washed and re-suspended in PBS. Then the cells were incubated with $2 \mu \mathrm{g} / \mathrm{ml} \mathrm{Rh} 123$ at $37^{\circ} \mathrm{C}$ for 20 min with gentle shaking. The samples were then analyzed by flow cytometry. The percentage of cells exhibiting a low level of Rh 123 uptake represented $\Delta \psi$ m loss. Histograms were analyzed using FCS Express V3.

\section{Determination of intracellular ROS}

Intracellular reactive oxygen species (ROS) production was detected through measuring the fluorescence intensity of dichlorofluorescein (DCF) as described by our previous papers [17]. 2, 7-DCFdiacetate (DCFH-DA), DCFH-DA is a non-fluorescent cell-permeant compound. Once inside the cell, it is cleaved by endogenous esterases and thereby trapped within the cell. The de-esterified product becomes fluorescent compound DCF on oxidation by ROS. At $1 \mathrm{~h}$ after different treatment, cells were harvested, washed and re-suspended in PBS, then incubated with $10 \mu \mathrm{M}$ DCFH-DA at $37{ }^{\circ} \mathrm{C}$ for 30 min with gentle shaking. Then the samples were monitored by flow cytometry. Histograms were analyzed using FCS Express V3 software.

\section{Statistical analysis}

The SPSS 16.0 software (SPSS Inc.) was used for the statistical analysis. Values are expressed as the mean \pm the standard error of the means (S.E.M) of three samples from three independent experiments. Statistical comparisons were made using the Student's t-test and one-way analysis of variance (ANOVA) followed by Tukey's test. Statistically significant differences were set at $\mathrm{p}<0.05$ or $\mathrm{p}<0.01$. 
Fig. 1. The time course for PpIX uptake in U937 cells. Cells were incubated with PpIX at a seriers of concentration for different time. At each time point, the autofluorescence of intracellular PpIX was measured by flow cytometry, and the mean fluorescence intensities were recorded. Data are presented as mean \pm S.D. from three independent experiments.

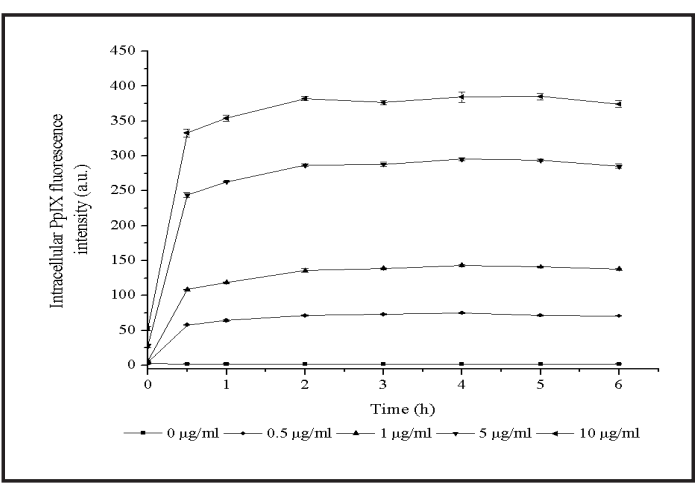

Fig. 2. Sub-cellular localization of PpIX in U937 cells. After incubation with $2 \mu \mathrm{g} / \mathrm{ml}$ PpIX (red channel) for $2 \mathrm{~h}$, cells were stained with MTG (green channel). After co-loading, cells were visualized by inverted confocal laser scanning microscope.

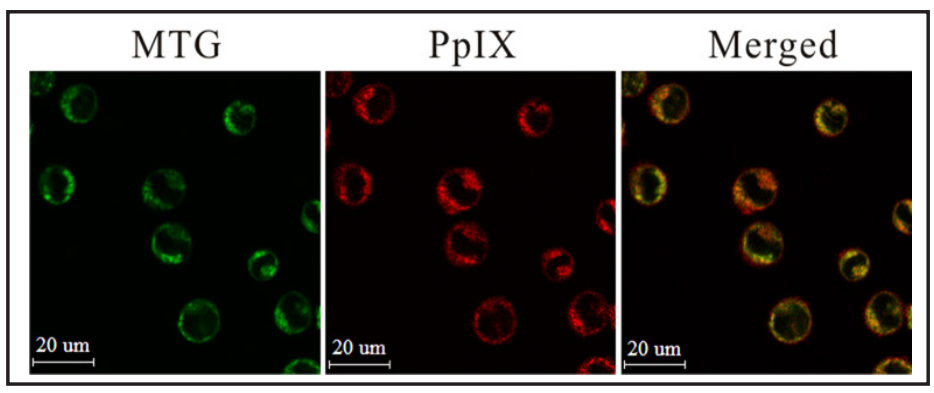

\section{Results}

Cellular uptake and localization of PpIX

Intracellular PpIX accumulation was determined by flow cytometry. As shown in Fig.1, the uptake process of PpIX was a dynamic change trend. Intracellular PpIX content rapidly increased at the first 0.5 hours when U937 cells were co-incubated with various initial concentration of PpIX $(0,0.5,1,5,10 \mu \mathrm{g} / \mathrm{ml})$ and reached a relatively high level at 2 hours after incubation. The result suggested that 2 hours may be the optimal incubation time of PpIX with U937 cells for the follow-up ultrasonic irradiation treatment.

After 2 hours incubation of PpIX, cells were co-loaded with mitochondria probe MTG. The Fig. 2 displayed that PpIX labeling pattern corresponded well with MTG, The co-staining images of PpIX and MTG showed a good over-lapping of both fluorescent signals indicating a higher concentration of PpIX in mitochondria in U937 cells, where PpIX mainly accumulated.

\section{Cytotoxicity induced by PpIX combined with ultrasound}

The cytotoxicity efficacy of PpIX combined with ultrasound on U937 cells was measured by MTT assay. As shown in Fig. 3, at $4 \mathrm{~h}$ post treatment the cell viability rate of PpIX group and Us group was $80.31 \%$ and $85.47 \%$ ( $<<0.05$ versus control group), respectively, indicating that PpIX alone and ultrasound alone all showed certain inhibitory effect on U937 cells under our experimental condition. Obviously, cell viability was significantly inhibited after treated by ultrasound plus $2 \mu \mathrm{g} / \mathrm{ml}$ PpIX, the viability rate declined to $52.25 \%$ ( $<<0.01$ versus other groups), suggesting an enhancement of cytotoxicity compared PpIX or ultrasound alone.

\section{Morphological changes of cells induced by PpIX combined with ultrasound}

The nuclei morphological changes were detected through DAPI staining at $4 \mathrm{~h}$ after treatment. Both the fluorescent image and the corresponding phase-contrast image were captured. As shown in Fig. 4, the nuclei of control cells appeared to be slightly blue and homogeneous fluorescence, normal growth state was shown with intact cell plasma membrane in phase contrast. Compared with control group, cells in Us group had no visible nuclei changes, displaying a weak well-distributed fluorescence with integrated morphology; cells in PpIX group showed slight enhanced DAPI staining while intact the phase contrast 
Fig. 3. The viability percentage of U937 cells at 4 $\mathrm{h}$ post different treatment. Data are means \pm S.D. of three independent experiments. ${ }^{*} \mathrm{p}<0.05$ and ${ }^{* *} \mathrm{p}<0.01$ versus the untreated control. $\# \# \mathrm{p}<0.01$ versus the PpIX alone and ultrasound alone groups.

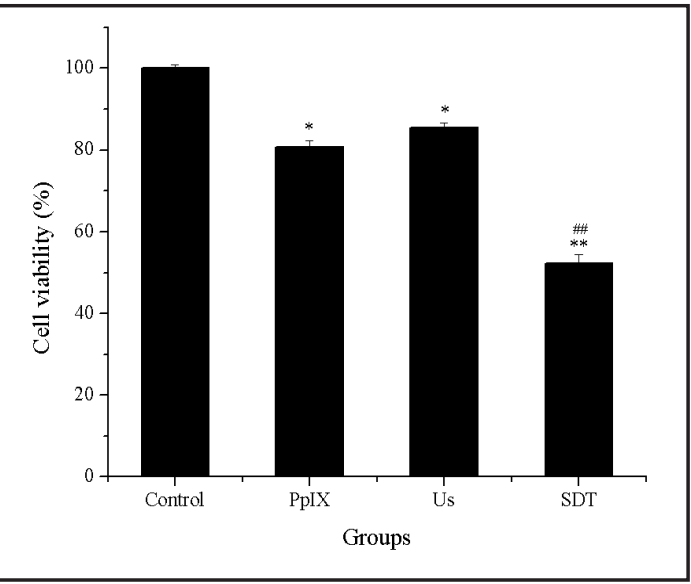

Fig. 4. Observation of morphological changes of U937 cells at $4 \mathrm{~h}$ post treatment. Cells in different groups were observed under fluorescent microscope. DAPI staining (above) and representative phase contrast (below) images were shown, respectively.

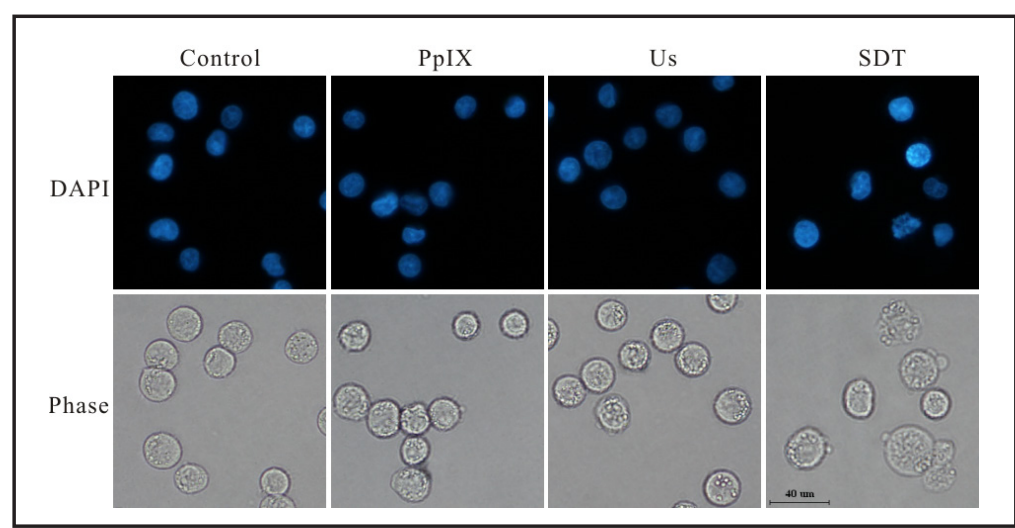

Fig. 5. DNA fragmentation analysis of U937 cells at $4 \mathrm{~h}$ after treatment. Cells were harvasted and re-suspended in Hanks' buffer containing $5 \mu \mathrm{g} / \mathrm{ml} \mathrm{PI}$, then permeabilized by freeze-thawing using liquid nitrogen. The samples were measured by flow cytometry, and the data were analyzed using FCS Express V3 software. Data shown are representative of three independent experiments.
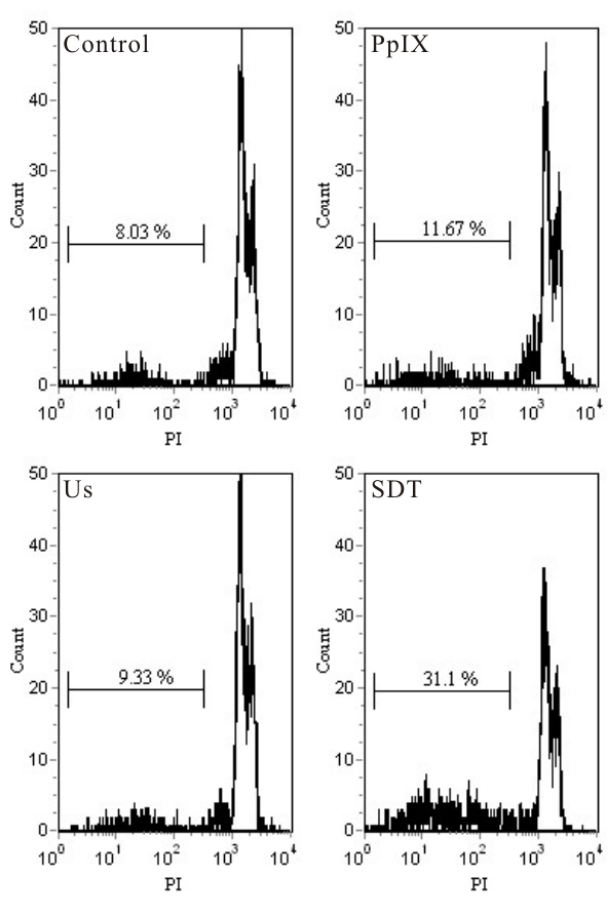

image displayed normal round morphology. Cells in SDT group clearly were seriously damaged with nuclear condensation with more enhancing DAPI staining and altered nuclear morphology were observed, and the phase images indicated cells were swollen. 

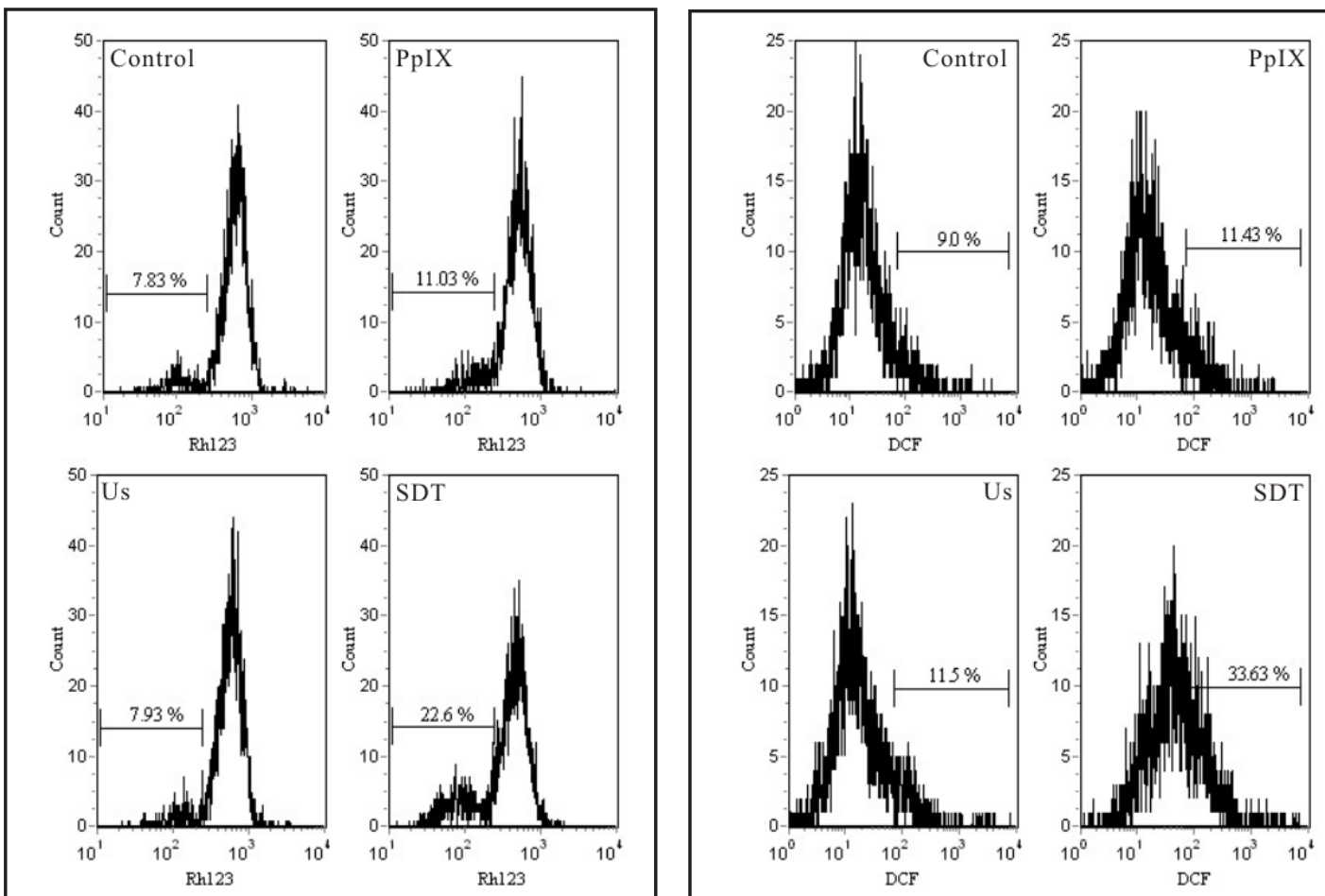

Fig. 6. Detection of mitochondrial membrane potential in U937 cells. At $2 \mathrm{~h}$ after treatment, cells were stained with $2 \mu \mathrm{g} / \mathrm{ml} \mathrm{Rh} 123$ and detected by flow cytometry. The data were analyzed using FCS Express V3 software. Data shown are representative of three independent experiments.
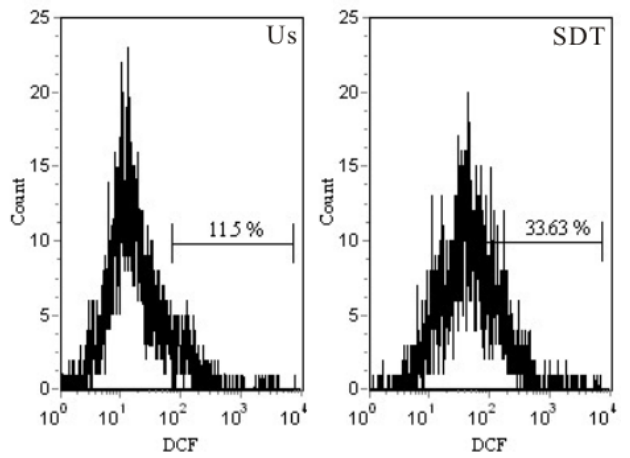

Fig. 7. Measurement of intracellular ROS in U937 cells. At $1 \mathrm{~h}$ post-treatment, cells were labeled with DCFH-DA and the fluorescence intensity of the oxidized product DCF in individual cells was detected by flow cytometry. Data shown are representative of three independent experiments.

\section{Analysis of DNA fragmantation}

As described in the Materials and Methods section, the DNA fragmentation was detected by flow cytometry.at $4 \mathrm{~h}$ after treatment. Fig. 5 showed the rate of DNA fragmentation in control group is $8.03 \%$, and those of PpIX group and Us group were $11.67 \%$ and $9.33 \%$, respectively. But in the SDT group, the level of DNA fragmentation significantly increased to $31.1 \%$. The results implied that PpIX-mediated SDT markedly induced DNA damage of U937 cells compared with PpIX or ultrasound alone.

\section{Mitochondrial damage assessment}

Rh123 staining combined with flow cytometry was used to evaluate the $\Delta \psi \mathrm{m}$ change to reflect the mitochondrial damage induced by PpIX-mediated SDT. The data in Fig. 6 showed that compared with the control cell $(7.83 \%)$, the percentage of cells exhibiting low level of Rh 123 uptake was up to $22.60 \%$ at $2 \mathrm{~h}$ post SDT treatment, while PpIX and ultrasound alone could not cause significant $\Delta \psi \mathrm{m}$ loss in U937 cells (11.03\% and $7.93 \%$, respectively).

\section{Production of intracellular ROS detection}

In this study, intracellular ROS production after different treatment was monitored through measuring the conversion of non-fluorescent DCFH-DA to fluorescent DCF using flow cytometry. Fig. 7 displayed that at $1 \mathrm{~h}$ post treatment, only $9.00 \%$ of cells in control group showed bright DCF fluorescence; In the PpIX group and ultrasound group, the percentage of cells with higher DCF fluorescence was about $11.43 \%$ and $11.50 \%$, respectively. But in the 


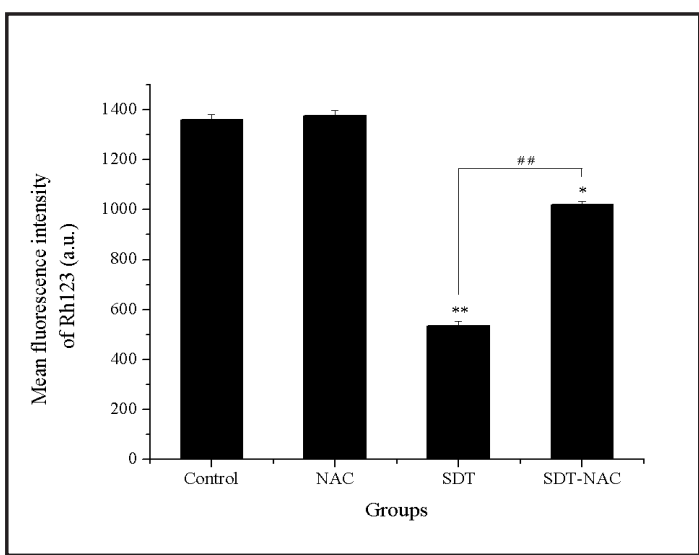

Fig. 8. Effect of the ROS scavenger NAC on SDT induced mitochondria membrane potential loss in U937 cells. At $2 \mathrm{~h}$ after treatment, cells were stained with $2 \mu \mathrm{g} / \mathrm{ml} \mathrm{Rh} 123$ and detected by flow cytometry. The fluorescence intensity of Rh123 shown are representative of three independent experiments. ${ }^{*} p<0.05$ and ${ }^{* *} p<0.01$ versus the untreated control. $\# \# \mathrm{p}<0.01$ versus the SDT group.

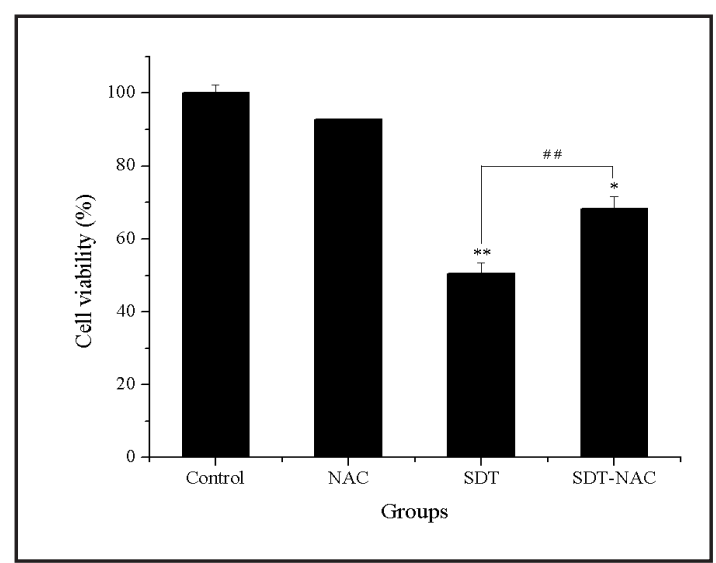

Fig. 9. Effect of the ROS scavenger NAC on SDT induced cytotoxicity in U937 cells. Cell viability was determined following exposure using MTT assay. ${ }^{*} \mathrm{p}<0.05$ and ${ }^{* *} \mathrm{p}<0.01$ versus the untreated control. $\# \# p<0.01$ versus the SDT group.

SDT group, the rate of cells exhibiting higher DCF fluorescence was up to $33.63 \%$, indicating PpIX mediated-SDT significantly enhanced the production of ROS.

We co-administrated a special ROS scavenger NAC to further investigate the role of ROS in SDT-induced cell damage in U937 cells. The results in Fig. 8 revealed that the fluorescence intensity of Rh 123 in the SDT sample pre-treated by NAC (the SDT-NAC group) was much higher than those of SDT group; Moreover, MTT assay revealed that decreased cell viability caused by SDT was obviously rescued by NAC (Fig. 9). These results indicated that ROS might be one of the factors which caused mitochondria damage, and it played an important role in the process of PpIX-mediated SDT killing U937 cells.

\section{Discussion}

It is well known that SDT can cause immediate sonolysis and delayed cell apoptosis by both direct mechanical stress and indirect chemical reaction $[2,6,18]$. Numerous studies demonstrated the ultrasound could induce apoptosis in a variety of cell types combined with an array of different sensitizers. In 2000, Ashush reported that ultrasound could trigger cell apoptosis in leukemia cells [19]. Subsequently, some studies indicated that lowintensity ultrasound combined with sonosensitizers could synergistically enhance apoptosis in leukemia cells. Changes caused by SDT in the malignant cell such as cell membrane destruction, mitochondria damage, chromatin condensation and ROS overload might be important factors that inhibited the tumor cell growth and even induced cell death [19-21]. Until now, the synergistic killing effect of SDT on leukemia cells is still unclear. Based on these earlier works, this study was to investigate the biological response of human leukemia U937 cells to PpIX mediated SDT treatment.

The effects of SDT depend mainly on the concentration and localization of sonosensitizer accumulated in the tumor cells. Sonosensitizer can enhance the ultrasonically induced cell damage in a concentration-dependent manner; the localization of sonosensitizer is potentially important for SDT because of the very short lifetime and very short diffusion distance of some radical products derived from the sonosensitizer during sonodynamic treatment process, 
which determines the localization of the primary damage $[22,23]$. In this study, the cellular uptake of PpIX and its intracellular localization in U937 cells were firstly investigated. The data in Figure 1 showed that PpIX accumulated in U937 cells quickly and sustained at a relatively high level at $2 \mathrm{~h}$ of incubation, and it mainly localized in the mitochondria of U937 cell (Fig. 2). Previous reports have shown that under physiological conditions, mitochondria accumulate PpIX for haem synthesis, and this process is regulated by many factors [24, 25]. Studies suggest that PpIX has a high affinity for peripheral benzodiazepine receptor on the outer membrane of mitochondrial [23]. That may be one of reasons why PpIX accumulation mainly localized in the mitochondria. In U937 cells, we found that PpIX mainly accumulated in the mitochondria, which is similar to other related studies.

In preliminary experiments, we also confirmed that PpIX decreased cell survival rate in a dose-dependent way in U937 cells (data not shown). Therefore, $2 \mu \mathrm{g} / \mathrm{ml}$ PpIX was selected in the present experiment, as the cell survival rate was approximately $80 \%$ when cells were treated by $2 \mu \mathrm{g} / \mathrm{ml} \mathrm{PpIX} \mathrm{(Fig.} \mathrm{3).} \mathrm{The} \mathrm{cell} \mathrm{viability} \mathrm{rate} \mathrm{was} \mathrm{assessed} \mathrm{by} \mathrm{MTT} \mathrm{assay} \mathrm{at} 4 \mathrm{~h}$ post SDT treatment. Significantly, cell damage was remarkably enhanced by PpIX plus ultrasound, whereas ultrasound alone did not show serious damage effect on U937 cells (cell survival rate was $85.47 \%$ in ultrasound alone group versus control group).

The morphology change and nucleus damage were studied by phase contrast and fluorescence microscope (Fig. 4). At $4 \mathrm{~h}$ post treatment, results obtained from DAPI staining showed enhanced blue fluorescence with condensed chromatin; simultaneously, the cells treated by SDT exhibited obvious cell swelling or necrosis with broken cell membrane under phase microscopy. But PpIX or ultrasound alone had little effect on U937 cells under experimental condition. Moreover, as shown in Figure 5, more significant DNA fragmentation was detected in SDT by flow cytometry (31.1\% versus $8.03 \%$ in control group, $11.67 \%$ in PpIX group and $9.33 \%$ in ultrasound group). The typical apoptotic characteristics of nucleus and high level of DNA fragmentation in U937 cells treated by SDT prompted that apoptotic death would happen when U937 cell were exposed to SDT treatment. A lot of studies on the anti-tumor effect of SDT hypothesize that the extrinsic death receptor pathway and the mitochondria-initiated pathway, which participate in cell apoptosis, might be involved in the malignant cells response to SDT [14, 26, 27]. More details about apoptosis in U937 cells under SDT treatment are still in further study.

As mentioned above, we confirmed that PpIX mainly accumulated in mitochondria of U937 cells. Lin et al found that PpIX overload disrupts mitochondrial dynamics in hepatocytes [28]. It is presumed that PpIX would mediate mitochondria damage under ultrasound irradiation. Therefore, the mitochondrial membrane potential changes $(\Delta \psi \mathrm{m})$ of U937 cells were monitored after sonication. As shown in Fig. 6, an initial drop in $\Delta \psi \mathrm{m}$ was detected at $2 \mathrm{~h}$ post treatment, indicating that the mitochondria were seriously damaged after SDT treatment. This result was consistent with our previous studies, suggesting the mitochondria may be one of the most effective damaging sites in SDT treatment $[13,20]$. As the major energy generators, mitochondria mediated apoptosis occurs in response to a wide range of stimuli, and the decrease of $\Delta \psi \mathrm{m}$ as a result of mitochondria depolarization in association with apoptosis appears to be a common event.

ROS are highly reactive molecules that originate mainly from the mitochondrial electron transport chain, and damaged mitochondria stimulate increased ROS production, which may subsequently aggravate mitochondria damage [29]. It is known that some ROS products derived from the sonosensitizer during sonodynamic treatment process, and they are important factors of cell death regulation [20, 30,31]. In our experiment, the generation of intracellular ROS was detected. As shown in Figure 7, the intracellular ROS in SDT group displayed a rapid generation, whereas there was no occurrence in the control, PpIX and ultrasound alone groups. Moreover, pre-treatment with NAC, a scavenger of ROS, the descent of Rh 123 uptake caused by SDT was inhibited, and the cell killing effect of SDT was also inhibited. These results suggested that intracellular excessive ROS generation was induced by PpIX-SDT treatment, and an oxidative stress mechanism was involved in response to sonochemical effect in U937 cells. ROS can modulate wide varieties of biological processes 
by coupling the signals at the cell surface into long-term changes in gene expression through multiple and complex signaling pathways [32]. During response to PpIX-mediated SDT treatment, excessive ROS generation usually occurs in various tumor cells, and plays an importantly role in the tumor inhibition induced by SDT. Our results confirmed that in U937 cells, the level of ROS was increased significantly after SDT treatment, and the relationship between ROS and apoptotic death induced by SDT is worthy of in-depth investigation.

\section{Conclusion}

In conclusion, the results of this study evidenced that PpIX-mediated SDT could exert its anti-tumor effect by triggering apoptosis in U937 cells. In this process, seriously morphological change, nucleus damage, DNA fragment increase and mitochondria change were obviously detected. ROS generation was involved in cell death induced by SDT, but the concrete analysis of this mechanism still need further study.

\section{Disclosure Statement}

The authors declare no competing financial interests.

\section{Acknowledgement}

This work was supported by the National Natural Science Foundation of China (Nos. 81000999, 10904087), the Research Fund for the Doctoral Program of Higher Education of China (No. 20100202110006), the Fundamental Research Funds for the Central Universities (No.GK201302022), and the Hong Kong Scholars Program (No. XJ2013034).

\section{References}

1 Jin H, Zhong X, Wang Z, Huang X, Ye H, Ma S, Chen Y, Cai J: Sonodynamic effects of hematoporphyrin monomethyl ether on CNE-2 cells detected by atomic force microscopy. J Cell Biochem 2011;112:169-178.

2 Shibaguchi H, Tsuru H, Kuroki M, Kuroki M: Sonodynamic cancer therapy: a non-invasive and repeatable approach using low-intensity ultrasound with a sonosensitizer. Anticancer Res 2011;31:2425-2429.

3 Pan H, Ma X, Li M, Chen J, Jiang H: Ultrasound assistant chemotherapy may be a novel modality for solid tumors. Med Hypotheses 2009;73:526-527.

-4 Yu T, Wang Z, Mason TJ: A review of research into the uses of low level ultrasound in cancer therapy. Ultrason Sonochem 2004;11:95-103.

5 Rosenthal I, Sostaric JZ, Riesz P: Sonodynamic therapy--a review of the synergistic effects of drugs and ultrasound. Ultrason Sonochem 2004;11:349-363.

-6 Bai W, Shen E, Hu B: Induction of the apoptosis of cancer cell by sonodynamic therapy: A review. Chinese Journal of Cancer Research 2012;24:368-373.

7 Matthew T: The promise of sonodynamic therapy. Cancer Metastasis Review 2013;DOI 10.1007/s10555013-9461-5.

-8 Liu Q, Wang X, Wang P, Xiao L, Hao Q: Comparison between sonodynamic effect with protoporphyrin IX and hematoporphyrin on sarcoma 180. Cancer Chemother Pharmacol 2007;60:671-680.

-9 Belcher JD, Beckman JD, Balla G, Balla J, Vercellotti G: Heme degradation and vascular injury. Antioxid Redox Signal 2010;12:233-248.

10 Wang X, Wang P, Tong W, Liu Q: Comparison of pharmacokinetics, intracellular localizations and sonodynamic efficacy of endogenous and exogenous protoporphyrin IX in sarcoma 180 cells. Ultrasonics 2010;50:803-810. 


\section{Cellular Physiology $\quad$ Cell Physiol Biochem 2014;33:1186-1196 and Biochemistry \\ Li et al.: The Cytotoxic Effects of PpIX Combined with Ultrasound on U937 Cells}

11 Cheng J, Sun X, Guo S, Cao W, Chen H, Jin Y, Li B, Li Q, Wang H, Wang Z, Zhou Q, Wang P, Zhang Z, Cao W, Tian Y: Effects of 5-aminolevulinic acid-mediated sonodynamic therapy on macrophages. Int J Nanomedicine 2013, 8:669-676.

12 Guo S, Sun X, Cheng J, Xu H, Dan J, Shen J, Zhou Q, Zhang Y, Meng L, Cao W, Tian Y: Apoptosis of THP1 macrophages induced by protoporphyrin IX-mediated sonodynamic therapy. Int J Nanomedicine 2013;8:2239-2246.

13 Li Y, Wang P, Zhao P, Zhu S, Wang X, Liu Q: Apoptosis induced by sonodynamic treatment by protoporphyrin IX on MDA-MB-231 cells. Ultrasonics 2012;52:490-496.

-14 Liang L, Xie S, Jiang L, Jin H, Li S, Liu J: The Combined effects of hematoporphyrin monomethyl ether-SDT and doxorubicin on the proliferation of QBC939 cell lines. Ultrasound Med Biol 2013;39:146-160.

15 Neidle S: DNA minor-groove recognition by small molecules. Nat Prod Rep 2001;18:291-309.

16 Krysko DV, Vanden Berghe T, D'Herde K, Vandenabeele P: Apoptosis and necrosis: detection, discrimination and phagocytosis. Methods 2008;44:205-221.

17 Wang H, Wang X, Wang P, Zhang K, Yang S, Liu Q: Ultrasound Enhances the Efficacy of Chlorin e6-Mediated Photodynamic Therapy in MDA-MB-231 Cells. Ultrasound Med Biol 2013;39:1713-1724.

18 Miyoshi N, Sostaric JZ, Riesz P: Correlation between sonochemistry of surfactant solutions and human leukemia cell killing by ultrasound and porphyrins. Free Radic Biol Med 2003;34:710-719.

19 Ashush H, Rozenszajn LA, Blass M, Barda-Saad M, Azimov D, Radnay J, Zipori D, Rosenschein U: Apoptosis induction of human myeloid leukemic cells by ultrasound exposure. Cancer Res 2000;60:1014-1020.

20 Su X, Wang P, Wang X, Guo L, Li S, Liu Q: Involvement of MAPK activation and ROS generation in human leukemia U937 cells undergoing apoptosis in response to sonodynamic therapy. Int J Radiat Biol 2013;89:915-927

21 Zhao P, Liu Q, Wang P, Li T, Wang X, Su S, Zhang M: Autophagic and apoptotic response to sonodynamic therapy induced cell damage in leukemia 1210 cells in vitro. Cancer Biother Radiopharm 2011;26:209218.

-22 Ji Z, Yang G, Vasovic V, Cunderlikova B, Suo Z, Nesland JM, Peng Q: Subcellular localization pattern of protoporphyrin IX is an important determinant for its photodynamic efficiency of human carcinoma and normal cell lines. J Photochem Photobiol B 2006;84:213-220.

23 Pastorino JG, Simbula G, Gilfor E, Hoek JB, Farber JL: Protoporphyrin IX, an endogenous ligand of the peripheral benzodiazepine receptor, potentiates induction of the mitochondrial permeability transition and the killing of cultured hepatocytes by rotenone. J Biol Chem 1994;269:31041-31046.

24 Koller ME, Romslo I: Uptake of protoporphyrin IX by isolated rat liver mitochondria. Biochem J 1980;188:329-335.

25 Zeno S, Veenman L, Katz Y, Bode J, Gavish M, Zaaroor M: The 18 kDa mitochondrial translocator protein (TSPO) prevents accumulation of protoporphyrin IX. Involvement of reactive oxygen species (ROS). Curr Mol Med 2012;12:494-501.

26 Tang W, Liu Q, Wang X, Wang P, Cao B, Mi N, Zhang J: Involvement of caspase 8 in apoptosis induced by ultrasound-activated hematoporphyrin in sarcoma 180 cells in vitro. J Ultrasound Med 2008;27:645-656.

27 Wang X, Liu Q, Wang P, Zhang K, Tang W, Wang B: Enhancement of apoptosis by sonodynamic therapy with protoporphyrin IX in isolate sarcoma 180 cells. Cancer Biother Radiopharm 2008;23:238-246.

-28 Lin YH, Chang HM, Chang FP, Shrn CR, Liu CL, Mao WY, Lin CC, Lee HS, Shen CN: Protoporphyrin IX accumulation disrupts mitochondrial dynamics and function in ABCG2-deficient hepatocytes. FEBS Lett 2013;587:3202-3209.

29 Drose S: Differential effects of complex II on mitochondrial ROS production and their relation to cardioprotective pre- and postconditioning. Biochim Biophys Acta 2013;1827:578-587.

-30 Misik V, Riesz P: Free radical intermediates in sonodynamic therapy. Ann N Y Acad Sci 2000;899:335-348.

- 31 Yumita N, Iwase Y, Nishi K, Komatsu H, Takeda K, Onodera K, Fukai T, Ikeda T, Umemura S, Okudaira K, Momose Y: Involvement of reactive oxygen species in sonodynamically induced apoptosis using a novel porphyrin derivative. Theranostics 2012;2:880-888.

-32 Montero AJ, Jassem J: Cellular redox pathways as a therapeutic target in the treatment of cancer. Drugs 2011;71:1385-1396. 